Artículo

\title{
Crecimiento, rendimiento y calidad de chile poblano cultivado en hidroponía bajo invernadero
}

\author{
Brenda Nataly Hernández Hernández ${ }^{1}$ \\ Mario Alberto Tornero Campante ${ }^{1 \S}$ \\ Engelberto Sandoval Castro ${ }^{1}$ \\ María de las Nieves Rodríguez Mendoza² \\ Oswaldo Rey Taboada Gaytán ${ }^{1}$ \\ Benjamín V. Peña Olvera ${ }^{1}$
}

${ }^{1}$ Colegio de Postgraduados-Campus Puebla. Boulevard Forjadores núm. 205, Santiago Momoxpan, San Pedro Cholula, Puebla, México. CP. 72760. Tel. 222 2851442. (bn-hdz@hotmail.com; engelber@colpos.mx; toswaldo@colpos.mx; bpena@colpos.mx). ${ }^{2}$ Colegio de Posgraduados-Campus Montecillo. Carretera México-Texcoco km 36.5, Montecillo, Texcoco, Estado de México, México. CP. 56230. Tel. 595 9520200. (marinie@ colpos.mx).

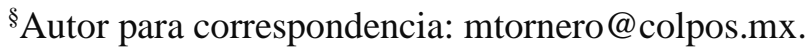

\section{Resumen}

El cultivo de chile poblano sembrado en suelo en el Alto Atoyac en Puebla se ha visto afectado por la presencia de enfermedades causadas por hongos, bacterias y nematodos, generando pérdidas en el rendimiento y reducción de la superficie de siembra. La producción bajo invernadero e hidroponía constituye una alternativa de solución a esta problemática, pues permite el desarrollo de cultivos en un ambiente controlado utilizando sustratos inertes, asegurando en mayor porcentaje la producción. El objetivo fue evaluar el crecimiento, rendimiento y calidad de fruto de dos variedades locales de chile poblano (Tlacotepec y Tlalancaleca) y una variedad comercial (San Luis), abastecidas nutrimentalmente mediante tres concentraciones de la solución nutritiva Steiner bajo invernadero e hidroponía con la finalidad de conocer su comportamiento agronómico y productivo. Se consideró un diseño de tratamientos factorial 3 × 3 en un diseño experimental completamente al azar. Las variables evaluadas fueron: altura de planta, grosor de tallo, número de hojas, número de bifurcaciones, número de flores, rendimiento y calidad de fruto. Los resultados mostraron que la variedad San Luis alcanzó el mayor rendimiento y calidad de fruto. En cuanto a las tres concentraciones de solución nutritiva, no se presentó efecto significativo en el rendimiento y el comportamiento agronómico de las variedades criollas en el sistema de sustrato, invernadero e hidroponía puede ser una opción para la producción de chile poblano en la región del Alto Atoyac.

Palabras clave: Capsicum annuиm L., agricultura protegida, solución nutritiva.

Recibido: junio de 2021

Aceptado: agosto de 2021 


\section{Introducción}

El chile (Capsicum annuum L.) es originario de México y de los cultivos más importantes a nivel mundial, es símbolo que da identidad a los mexicanos en diversas culturas y tiene gran impacto en la gastronomía nacional e internacional (Aguirre y Muñoz, 2015), sus frutos se consumen en fresco y en seco para proporcionar color, sabor y aroma a infinidad de platillos. México es productor de diversas variedades del género Capsicum, donde se cultivan alrededor de 158000 ha de chile verde con una producción promedio de 3.3 millones de toneladas anuales, convirtiéndolo en el segundo productor a nivel mundial después de China (SIAP, 2019).

En Puebla se produce una gran diversidad de chiles, entre ellos, el chile poblano criollo por poseer una gran importancia económica, sociocultural y gastronómica (Rodríguez et al., 2007), éste se produce principalmente en la región del Alto Atoyac en el sistema tradicional a cielo abierto, los municipios que destacan en la producción de chile poblano son: San Martín Texmelucan, San Lorenzo Chiautzingo, San Salvador el Verde, San Felipe Teotlalcingo, San Matías Tlalancaleca, entre otros (SIAP, 2019).

No obstante, en los últimos años el cultivo ha sido afectado por diversos factores y problemas fitosanitarios, predominando las enfermedades en el suelo ocasionadas por hongos, bacterias y nematodos, responsables de la reducción del rendimiento y la superficie de siembra, lo que ha generado pérdidas económicas para los productores quienes no cuentan con paquetes tecnológicos adecuados para enfrentar dichos problemas (González-Pérez et al., 2004; Rodríguez et al., 2007; Pérez et al., 2017).

Ante esta problemática, es indispensable buscar sistemas alternativos para la producción de chile poblano, uno de ellos es la hidroponía que se caracteriza por no requerir de suelo como sistema biótico y de sostén para las plantas y ofrece la posibilidad de obtener altos rendimientos (Velasco et al., 2011; Sánchez-del-Castillo et al., 2014). En el sistema hidropónico se suministran los nutrientes que las plantas demandan a través de una solución nutritiva, una de las soluciones más empleadas para la producción de Capsicum annuиm es la solución nutritiva formulada por Steiner (Beltrán-Morales et al., 2016; Luna-Fletes et al., 2018; San Juan et al., 2019) dicha solución, debe tener la concentración de nutrimentos adecuada pues de ello dependerá el rendimiento y calidad de los frutos (San Juan et al., 2019).

Para conducir con éxito el cultivo de chile poblano en este sistema, es necesario realizar estudios enfocados en el manejo agronómico y la nutrición, pues de todos los factores que influyen sobre el crecimiento del cultivo, la nutrición vegetal es uno de los más determinantes (Alcántar-González et al., 2016). Existe información insuficiente relacionada con el manejo nutrimental en chile poblano cultivado bajo invernadero e hidroponía, en especial sobre variedades criollas o locales, tampoco hay una solución nutritiva específica para el cultivo pues las investigaciones se han enfocado primordialmente en el cultivo en suelo y a cielo abierto.

De ahí, que la pregunta de investigación esté relacionada con identificar el comportamiento agronómico de variedades criollas de chile poblano en sustrato, cuando son sometidas al sistema de producción en invernadero e hidroponía. Con base en lo anterior, el objetivo fue evaluar el crecimiento, rendimiento y calidad de fruto de dos variedades nativas de chile poblano (Tlacotepec 
y Tlalancaleca) y una variedad comercial (San Luis), abastecidas nutrimentalmente mediante tres concentraciones de la solución nutritiva Steiner, con la finalidad de conocer su comportamiento agronómico y productivo.

\section{Materiales y métodos}

La investigación se realizó en la unidad de agricultura protegida del Colegio de Postgraduados ubicada en San Agustín Calvario, San Pedro Cholula, Puebla, a 1903' 18' latitud norte y $98^{\circ} 20^{\prime}$ 59" de longitud oeste, con una altitud de 2166 m (INEGI, 2010), durante el periodo primaveraotoño 2017. Se evaluaron dos variedades locales de chile poblano de la región del Alto Atoyac, Puebla, identificadas como Tlacotepec y Tlalancaleca, provenientes de los municipios de Tlacotepec de José Manzo (19 $27^{\circ}$ '94" latitud norte y 98 49' 12" longitud oeste) y San Matías Tlalancaleca ( $19^{\circ} 17^{\prime} 30^{\prime \prime}$ latitud norte y $98^{\circ} 27^{\prime}$ 42” longitud oeste), también se evaluó la variedad San Luis de la empresa Caloro, dichos materiales son cultivados por los productores de la región en el sistema tradicional en campo.

La siembra se realizó en charolas de poliestireno de 200 cavidades, se utilizó turba (peat moss) marca Sunshine mezcla 3 como sustrato, la emergencia ocurrió 16 días después de la siembra. El trasplante se realizó 32 días después de la emergencia en bolsas de polietileno negro $(40$ x $40 \mathrm{~cm})$ con capacidad de $11 \mathrm{~kg}$, las cuales se llenaron con arena volcánica (tezontle rojo) con partículas tamaño igual o inferior a $2 \mathrm{~mm}$ de diámetro y en cada bolsa se colocaron dos plántulas a profundidad del cepellón. Durante los 14 días después del trasplante (ddt) se aplicó un riego diario con solución nutritiva diluida al 75\%, a partir del día 15 (ddt) se inició con la aplicación de los tratamientos.

El diseño experimental fue un arreglo factorial 3x3, los factores de estudio fueron variedades: Tlacotepec (TC), Tlalancaleca (TL) y Ancho San Luis (SL) y concentración de la solución nutritiva (SN) Steiner: 50, 75 y 100\%. Los tratamientos fueron distribuidos bajo un diseño completamente al azar con 12 repeticiones. La unidad experimental fue una bolsa con sustrato con dos plantas. La densidad de plantación fue de 4.2 plantas $\mathrm{m}^{-2}$.

La solución nutritiva utilizada fue la Universal propuesta por Steiner (1984), con 12, 1 y $7 \mathrm{me} \mathrm{L}^{-1}$ de $\mathrm{NO}_{3}{ }^{-}, \mathrm{H}_{2} \mathrm{PO}_{4}^{-}$y $\mathrm{SO}_{4}{ }^{2-}$ para aniones y 7,9 y 4 me $\mathrm{L}^{-1}$ de $\mathrm{K}^{+}, \mathrm{Ca}^{2+}$ y $\mathrm{Mg}^{2+}$ para cationes, además de: 2, 0.7, 0.02, 0.09, 0.05 y $0.04 \mathrm{mg} \mathrm{L}^{-1}$ de Fe, Mn, Cu, Zn, B y Mo para una solución de concentración $100 \%$. La solución se preparó con fertilizantes comerciales solubles y se aplicó mediante un sistema abierto. Para la concentración al $100 \%$ se utilizaron las siguientes fuentes de fertilizantes: $\mathrm{KNO}_{3}, \mathrm{Ca}\left(\mathrm{NO}_{3}\right)_{2} 4 \mathrm{H}_{2} \mathrm{O}, \mathrm{KH}_{2} \mathrm{PO}_{4}, \mathrm{MgSO}_{4} 7 \mathrm{H}_{2} \mathrm{O}, \mathrm{K}_{2} \mathrm{SO}_{4}$ y complejo comercial de micronutrientes Ultrasol Micro-Mix de SQM, las soluciones al 75 y $50 \%$ se prepararon proporcionalmente. Los riegos se aplicaron una vez al día de forma manual y variaron en función del crecimiento de las plantas, en promedio se aplicaron $250 \mathrm{ml} \mathrm{maceta}^{-1}$ en etapa de establecimiento y $1 \mathrm{~L} \mathrm{maceta}^{-1}$ en etapa de desarrollo, floración y fructificación. Para evitar la acumulación de sales en el sustrato se aplicó un riego con agua de pozo cada siete días.

Los caracteres evaluados en la planta fueron: altura, se midió con una cinta métrica desde la base del tallo hasta el último ápice foliar, grosor de tallo, se midió con un vernier digital a $2 \mathrm{~cm}$ del nivel del sustrato, número de bifurcaciones, número de hojas y número de flores, se determinaron 
contabilizándolos directamente del tallo principal, rendimiento, se registró el número de frutos y el peso total $(\mathrm{kg})$ al momento de la cosecha, caracterización de fruto, se determinó con la Norma Mexicana: NMX-FF-025-SCFI-2014 y se registró el largo de fruto con una regla graduada, ancho de fruto con un vernier digital y peso de fruto con una balanza electrónica. Las variables de crecimiento se registraron semanalmente a partir del primer día del trasplante hasta los $154 \mathrm{ddt}$, el rendimiento y calidad de fruto se registraron a los 97, 113, 127, 148 y 159 ddt.

Los datos obtenidos se sometieron a un análisis de varianza y prueba de comparación de medias de Tukey $(p \leq 0.05)$ con el programa Statistical Analysis System versión 9.0 (SAS Institute, 2002). Adicionalmente, a las variables de crecimiento se les aplicó el modelo no lineal NLIN propuesto por Hunt (2017), con el propósito de observar el ajuste de cada parámetro evaluado en invernadero, al aplicar un modelo matemático.

\section{Resultados y discusión}

\section{Crecimiento}

El análisis de varianza mostró para el factor variedad (VAR) diferencias altamente significativas en altura de planta (AP), grosor de tallo $(\mathrm{GT})$ y número de hojas $(\mathrm{NH})$, diferencias significativas en número de bifurcaciones (NB) y no significativas en el número de flores (NF). No se encontraron diferencias significativas por efecto de la concentración de la solución nutritiva (SN), solamente en NH las diferencias fueron altamente significativas (Cuadro 1).

Cuadro 1. Cuadrados medios y significancia estadística de las variables de crecimiento, en chile poblano cultivado en hidroponía e invernadero.

\begin{tabular}{|c|c|c|c|c|c|c|c|c|c|c|c|}
\hline \multirow{2}{*}{$\mathrm{FV}$} & \multirow{2}{*}{ GL } & AP & $\mathrm{Fc}$ & GT & $\mathrm{Fc}$ & NB & $\mathrm{Fc}$ & $\mathrm{NH}$ & $\mathrm{Fc}$ & $\mathrm{NF}$ & $\mathrm{Fc}$ \\
\hline & & \multicolumn{6}{|c|}{$154 \mathrm{ddt}$} & \multicolumn{2}{|c|}{$91 \mathrm{ddt}$} & \multicolumn{2}{|c|}{$42 \mathrm{ddt}$} \\
\hline SN & 2 & 242.8 & $0.9 \mathrm{~ns}$ & 0.7 & $2 \mathrm{~ns}$ & 55.7 & $1.3 \mathrm{~ns}$ & 387 & $5.6^{* *}$ & 7.6 & $2.8 \mathrm{~ns}$ \\
\hline VAR & 2 & 9955.4 & $37.6^{* *}$ & 163.4 & $480.3^{* *}$ & 297.6 & $6.8^{*}$ & 4241.2 & $61.1^{* *}$ & 22.3 & $8.3^{* *}$ \\
\hline $\mathrm{SN} * \mathrm{VAR}$ & 4 & 2217.6 & $8.4^{* *}$ & 0.9 & $2.6^{*}$ & 53.7 & $1.2 \mathrm{~ns}$ & 83.9 & $1.2 \mathrm{~ns}$ & 6.7 & $2.5^{*}$ \\
\hline Error & 99 & 264.5 & - & 0.3 & - & 43.6 & - & 69.4 & - & 2.7 & - \\
\hline $\mathrm{CV}$ & - & 9.9 & - & 4.7 & - & 15 & - & 7 & - & 20.4 & - \\
\hline
\end{tabular}

$\mathrm{FV}=$ fuente de variación; $\mathrm{GL}=$ grados de libertad; $\mathrm{Fc}=\mathrm{F}$ calculado; $\mathrm{SN}=$ solución nutritiva; $\mathrm{VAR}=$ variedad; $\mathrm{AP}=$ altura de planta; $\mathrm{GT}=$ grosor de tallo; $\mathrm{NH}=$ número de hojas; $\mathrm{NB}=$ número de bifurcaciones; $\mathrm{NF}=$ número de flores; $\mathrm{CV}=$ coeficiente de variación $(\%) ;{ }^{*},{ }^{* *}=$ significancia estadística al 0.05 y 0.01 de probabilidad; ns= no significativo.

De acuerdo con los resultados de la prueba de comparación de medias (Cuadro 2), en la variable AP no se encontraron diferencias significativas por la aplicación de la solución nutritiva, solo se encontraron diferencias significativas entre variedades. Esto indica que la aplicación de una concentración alta de solución nutritiva no necesariamente garantiza una mayor AP, pues como lo mencionan Magdaleno-Villar et al. (2006); San Martín-Hernández et al. (2012) generalmente, al aumentar la concentración de nutrientes la conductividad eléctrica (CE) tiende a elevarse y reduce la capacidad de absorción de agua y nutrientes, lo que ocasiona que el crecimiento de la planta se vea limitado. 
Cuadro 2. Comparación de medias de las variables de crecimiento en tres variedades de chile poblano, cultivadas con tres concentraciones de solución nutritiva.

\begin{tabular}{|c|c|c|c|c|c|}
\hline \multirow[t]{2}{*}{ Concentración de la SN (\%) } & $\mathrm{AP}(\mathrm{cm})$ & $\begin{array}{c}\mathrm{GT} \\
(\mathrm{mm})\end{array}$ & $\begin{array}{c}\text { NB por } \\
\text { planta }\end{array}$ & \multirow{2}{*}{$\begin{array}{c}\begin{array}{c}\text { NH por } \\
\text { planta }\end{array} \\
91 \mathrm{ddt}\end{array}$} & \multirow{2}{*}{$\begin{array}{c}\text { NF por } \\
\text { planta } \\
42 \mathrm{ddt}\end{array}$} \\
\hline & \multicolumn{3}{|c|}{$154 \mathrm{ddt}$} & & \\
\hline 50 & $160.7 \mathrm{a}$ & $12.3 \mathrm{a}$ & $43.1 \mathrm{a}$ & $115.7 \mathrm{~b}$ & $7.6 \mathrm{a}$ \\
\hline 75 & $165.6 \mathrm{a}$ & $12.5 \mathrm{a}$ & $45.5 \mathrm{a}$ & $118.2 \mathrm{ab}$ & 8 a \\
\hline 100 & $164.5 \mathrm{a}$ & $12.3 \mathrm{a}$ & $43.6 \mathrm{a}$ & $122.2 \mathrm{a}$ & $8.5 \mathrm{a}$ \\
\hline DHS & 9.1 & 0.3 & 3.7 & 4.7 & 0.9 \\
\hline \multicolumn{6}{|l|}{ Variedad } \\
\hline San Luis & $147.8 \mathrm{c}$ & $14.8 \mathrm{a}$ & $41.2 \mathrm{~b}$ & $106.2 \mathrm{~b}$ & $8.6 \mathrm{a}$ \\
\hline Tlacotepec & $162.2 \mathrm{~b}$ & $11.1 \mathrm{~b}$ & $44.1 \mathrm{ab}$ & $125.3 \mathrm{a}$ & $8.4 \mathrm{a}$ \\
\hline Tlalancaleca & 180.9 a & $11.2 \mathrm{~b}$ & $47 \mathrm{a}$ & $124.7 \mathrm{a}$ & $7.1 \mathrm{a}$ \\
\hline DHS & 9.1 & 0.3 & 3.7 & 4.7 & 0.9 \\
\hline
\end{tabular}

Medias con la misma letra en la misma columna son estadísticamente iguales (Tukey, $p \leq 0.05$ ); DHS= diferencia honesta significativa. $\mathrm{AP}=$ altura de planta; $\mathrm{GT}=$ grosor de tallo; $\mathrm{NH}=$ número de hojas; $\mathrm{NB}=$ número de bifurcaciones; $\mathrm{NF}=$ número de flores; $\mathrm{SN}=$ solución nutritiva.

Por otra parte, en la Figura 1 se presentan las curvas de crecimiento ajustadas para AP, donde puede observarse que los valores más altos se obtuvieron en el tratamiento TL75\% (173.9 cm), seguido del tratamiento TC75\% $(151.4 \mathrm{~cm})$ y SL50\% $(138.8 \mathrm{~cm})$. Toledo-Aguilar et al. (2011) reportaron valores de altura de planta en 49 variedades nativas de chile poblano cultivadas a cielo abierto, que variaron de 38 hasta $57 \mathrm{~cm}$, las variedades evaluadas en este estudio presentaron una altura tres veces mayor debido a las condiciones en las que fueron cultivadas, lo cual, coincide con lo encontrado por Beltrán-Morales et al. (2016), quienes obtuvieron valores altos en altura de planta en chile jalapeño producido bajo invernadero en contraste con las cultivadas en campo.

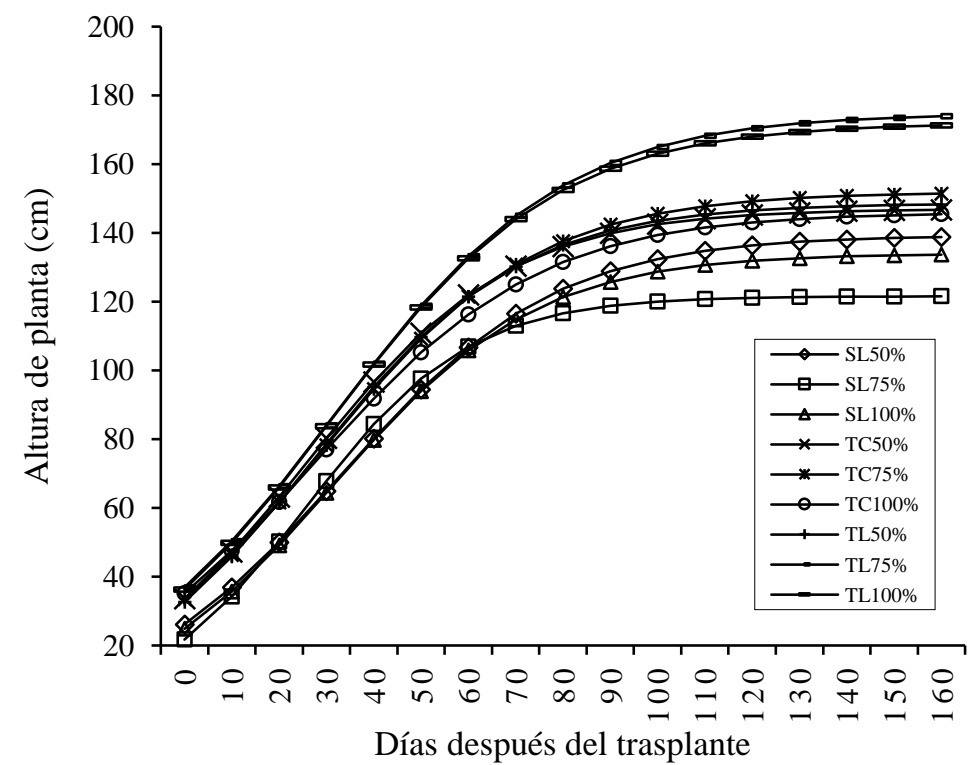

Figura 1. Dinámica de crecimiento de altura de planta en tres variedades de chile poblano, cultivadas con tres concentraciones de la solución nutritiva Steiner. 
Por lo regular, en condiciones controladas de invernadero si la planta se somete a una baja intensidad lumínica los entrenudos de los tallos se alargan dando como resultado una mayor altura de planta, por esta razón uno de los factores que también debe considerarse en la producción de Capsicum es la radiación solar pues influye directamente en el crecimiento del cultivo (HernándezVerdugo et al., 2015; Alemán et al., 2018).

En grosor de tallo, el análisis de varianza mostró diferencias significativas para la interacción VAR x SN (Cuadro 1), el GT se vio favorecido en la variedad San Luis (15 mm) comparado con las variedades locales donde se obtuvo un grosor de tallo promedio de $11 \mathrm{~mm}$ (Cuadro 2). Los datos obtenidos son semejantes a los reportados por Elizondo-Cabalceta y Monge-Pérez (2017) quienes obtuvieron valores para grosor de tallo entre 12.5 y $15.8 \mathrm{~mm}$ en 12 genotipos de chile dulce cultivados bajo condiciones de invernadero; por otra parte, Moreno et al. (2011) reportaron en 12 variedades comerciales de pimiento morrón cultivados bajo invernadero e hidroponía grosores de tallo que variaron de los 14 a los $16 \mathrm{~mm}$.

En la Figura 2, claramente se puede observar que los tratamientos sobresalientes fueron SL100\%, SL50\% y SL75\%, en contraste con las variedades locales las cuales presentaron valores inferiores, tal es el caso del TC50\%; no obstante, coinciden con el intervalo de valores obtenidos para grosor de tallo de 8.3 a $12.6 \mathrm{~mm}$ en plantas de chile cultivadas bajo condiciones protegidas (Moreno et al., 2014).

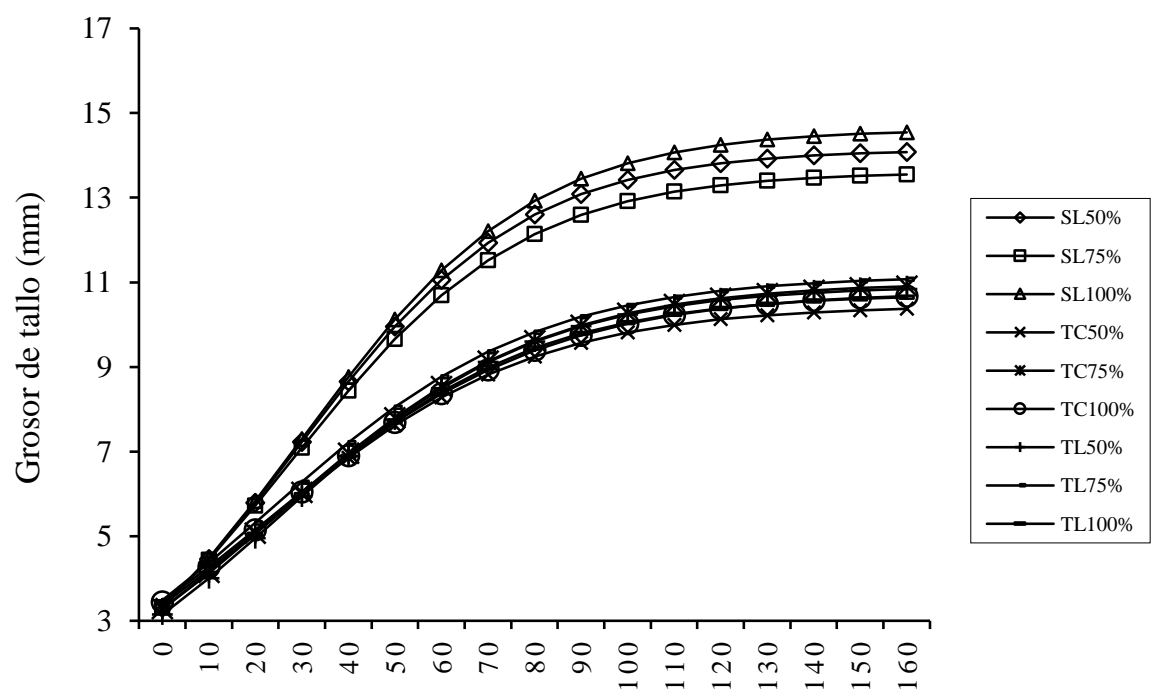

Días después del trasplante

Figura 2. Dinámica de crecimiento de grosor de tallo en tres variedades de chile poblano, cultivadas con tres concentraciones de la solución nutritiva Steiner.

En NH se reportan los resultados a los 91 ddt, debido a que los máximos valores para esta variable se registraron durante este muestreo. Los factores VAR y SN afectaron significativamente esta variable Cuadros 1 y 2, se encontró que la respuesta de las variedades de chile poblano a la concentración de la solución nutritiva fue lineal, a mayor concentración de la solución nutritiva el NH también se incrementó. Como se indica en la (Figura 3), los tratamientos que acumularon un mayor NH fueron TC100\%, TC75\%, TL100\% y TL75\%, el menor número de hojas corresponde al tratamiento SL75\%. 


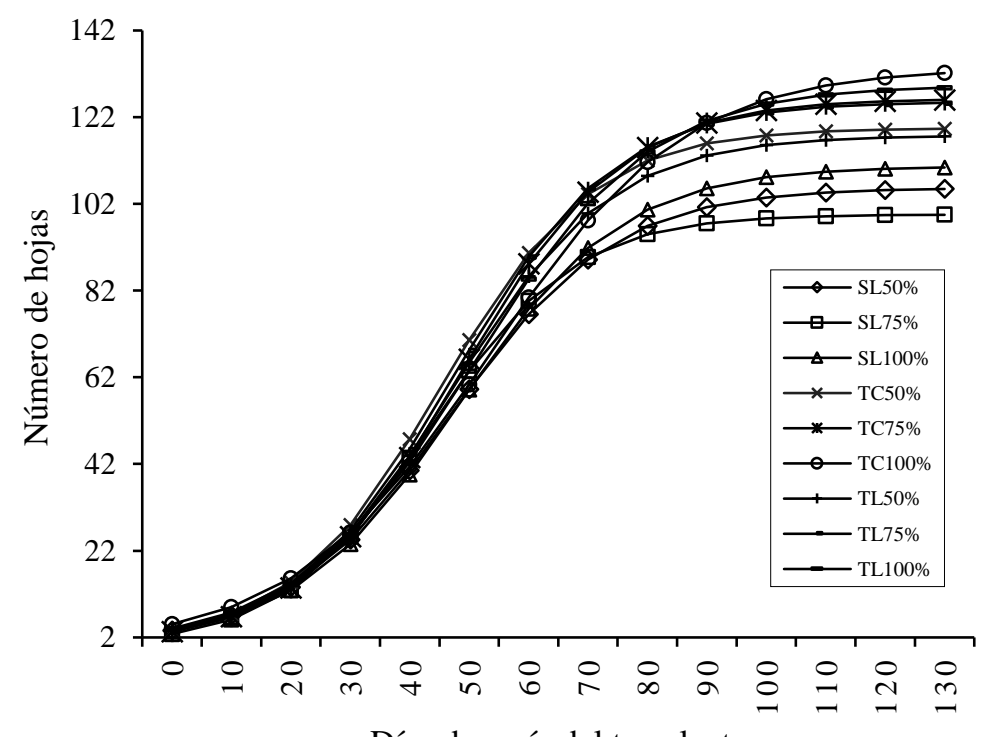

Días después del trasplante

Figura 3. Dinámica de crecimiento del número de hojas en tres variedades de chile poblano, cultivadas con tres concentraciones de la solución nutritiva Steiner.

Estos resultados son similares a los publicados por Fawzy et al. (2012) en C. annuum cv 'California Wonder' quienes observaron un mayor crecimiento vegetativo en las plantas con la aplicación de una solución nutritiva concentrada al 100\% de fertilizante químico. Además, el índice de área foliar en plantas de chile poblano cultivadas en condiciones de invernadero indica que una mayor cantidad de follaje influye significativamente en la captura de luz y $\mathrm{CO}_{2}$, pues al absorber una mayor cantidad de radiación fotosintéticamente activa se incrementa la producción foliar y la síntesis de compuestos esenciales para el desarrollo de la planta (Berrospe-Ochoa et al., 2015; Mendoza-Pérez et al., 2017).

La variable número de bifurcaciones (NB) fue diferente en las tres variedades (Cuadro 1), pero no hubo efecto por la solución nutritiva. En la variedad Tlalancaleca se obtuvo un valor superior para dicha variable (47 bifurcaciones), en comparación con la variedad Tlacotepec (44 bifurcaciones) y la variedad San Luis (41 bifurcaciones). Esta respuesta puede apreciarse en la Figura 4, en donde sobresalen los tratamientos TL75\%, TL100\% y TC75\% en contraste con los tratamientos SL50\% y SL75\%, tal como sucedió en AP dado que el NB está directamente relacionado con AP, pues a mayor altura de planta el número de bifurcaciones también es mayor y por consecuencia, podría esperarse que el número de frutos por planta también se incremente ya que en cada bifurcación, las plantas pueden desarrollar una flor que puede convertirse en un fruto potencial (Ponce et al., 2012).

Para NF se presentan los resultados a los 42 ddt debido a que en esta etapa las plantas de chile poblano alcanzaron su máxima expresión en esta variable. El NF mostró diferencias estadísticas significativas entre las variedades evaluadas, pero no por la aplicación de la solución nutritiva Steiner (Cuadro 1). 


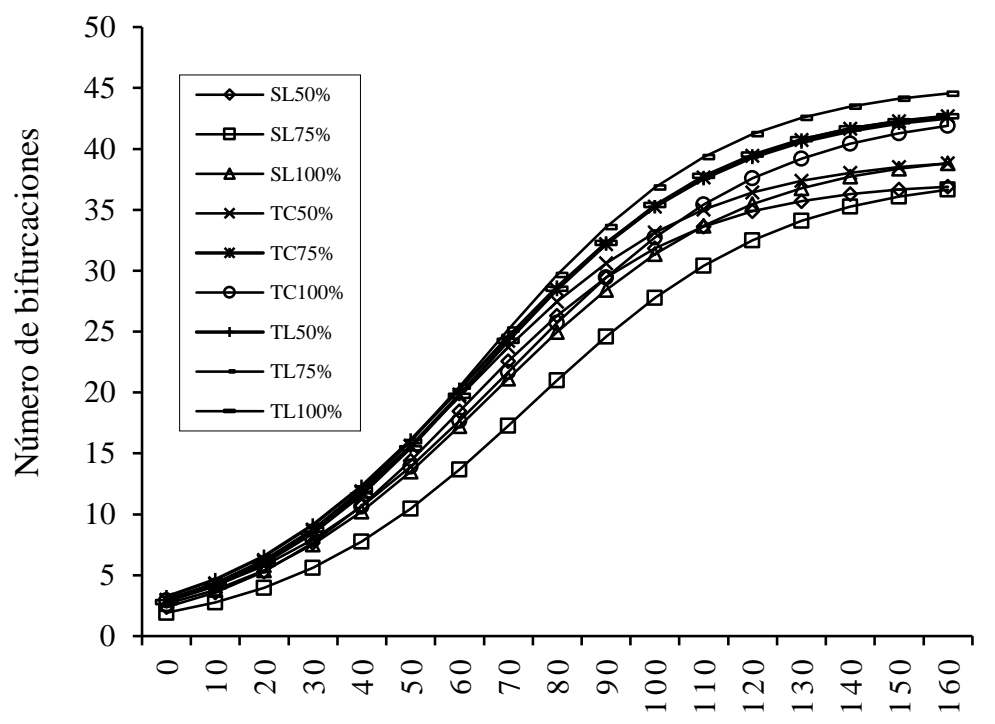

Días después del trasplante

Figura 4. Dinámica de crecimiento del número de bifurcaciones en tres variedades de chile poblano, cultivadas con tres concentraciones de la solución nutritiva Steiner.

En la Figura 5, puede observarse que a lo largo del ciclo de cultivo en los diferentes muestreos realizados se presentó un incremento y una disminución en el número de flores, esto debido a que las plantas presentaron aborto de flores y muchas de las flores contabilizadas no se convirtieron en fruto, por lo anterior, el crecimiento en esta variable no fue lineal. Reséndiz-Melgar et al. (2010), mencionan que las plantas de chile tienen crecimiento simpódico y en cada bifurcación se producen flores generalmente solitarias.

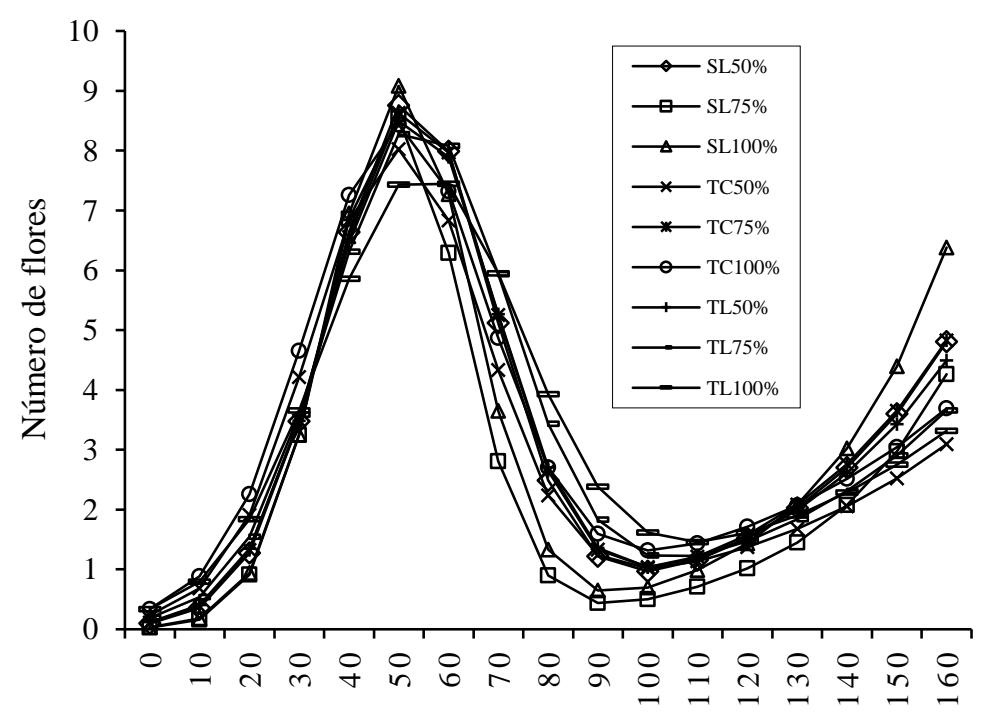

Días después del trasplante

Figura 5. Curvas de crecimiento de número de flores, en tres variedades de chile poblano cultivadas con tres concentraciones de solución nutritiva. 
Si las plantas se dejan crecer libremente, las primeras seis a 12 flores amarran fruto, pero la alta demanda de asimilados para su rápido crecimiento ocasiona aborto de un alto porcentaje de las flores generadas subsecuentemente. Los principales agentes causales de la caída de flores están asociados con altas temperaturas, baja intensidad de la radiación, presencia de fruto en la etapa de crecimiento rápido y diversos agentes bióticos (Moreno et al., 2011; López-Gómez et al., 2017).

\section{Rendimiento}

En esta variable no se presentaron diferencias estadísticas significativas por la aplicación de los tratamientos (Cuadro 3), las diferentes concentraciones de la SN no tuvieron efecto en el rendimiento. En cambio, en las variedades evaluadas hubo diferencias significativas como se puede observar en los (Cuadros 3 y 4), donde el rendimiento fue estadísticamente mayor en la variedad comercial San Luis (1.42 $\mathrm{kg}$ planta $\left.^{-1}\right)$ seguido de las variedades Tlalancaleca $\left(1.05 \mathrm{~kg} \mathrm{planta}^{-1}\right) \mathrm{y}$ Tlacotepec $\left(0.99 \mathrm{~kg} \mathrm{planta}^{-1}\right)$.

El rendimiento promedio reportado para el cultivo de chile poblano producido en campo varía de $1.4 \mathrm{t} \mathrm{ha}^{-1}$ a $3.2 \mathrm{t} \mathrm{ha}^{-1}$ (Velásquez-Valle et al., 2014; Pérez et al., 2017) y según los resultados obtenidos en esta investigación se pueden llegar a obtener rendimientos de hasta $4 \mathrm{~kg} \mathrm{~m}^{-2}$ con variedades nativas de la región y de $6 \mathrm{~kg} \mathrm{~m}^{-2}$ con variedades comerciales, datos semejantes a los reportados por Mendoza-Pérez et al. (2017) en el cultivo de chile poblano variedad Capulín, Ramos-Gourcy y De Luna-Jiménez (2006) en tres variedades de chile ancho y Aguilar et al. (2005) en chile ancho San Luis.

Cuadro 3. Análisis de varianza de rendimiento y número de frutos, en chile poblano cultivado en hidroponía e invernadero.

\begin{tabular}{cccccccccc}
\hline \multirow{2}{*}{ FV } & & \multicolumn{3}{c}{ Rendimiento $\left(\mathrm{kg} \mathrm{planta}^{-1}\right)$} & & \multicolumn{3}{c}{ Número de frutos por planta } \\
& & CM & Fc & Significancia & & CM & Fc & Significancia \\
\hline Solución nutritiva (SN) & 2 & 0.06 & 1.72 & 0.1855 & & 61.2 & 2.26 & 0.1103 \\
Variedad (VAR) & 2 & 1.95 & 53.65 & $<0.0001$ & & 393.7 & 14.55 & $<0.0001$ \\
SN*VAR & 4 & 0.05 & 1.24 & 0.2998 & & 95.5 & 3.53 & 0.0102 \\
Error & 88 & 0.04 & - & - & & 27.1 & - & - \\
CV & - & 16.59 & - & - & & 22.2 & - & -
\end{tabular}

$\mathrm{FV}=$ fuente de variación; $\mathrm{GL}=$ grados de libertad; $\mathrm{CM}=$ cuadrados medios; $\mathrm{Fc}=\mathrm{F}$ calculada $\mathrm{SN}=$ solución nutritiva; $\mathrm{VAR}=$ variedad; $\mathrm{CV}=$ coeficiente de variación $(\%) ; \mathrm{Sig}=$ significancia estadística al 0.05 y 0.01 de probabilidad.

Para la variable número de frutos se encontraron diferencias significativas y altamente significativas $(p \leq 0.05)$ entre las tres variedades evaluadas de chile poblano Cuadro 3, el mayor número de frutos se obtuvo con las variedades Tlacotepec y Tlalancaleca (Cuadro 4). Las concentraciones aplicadas (50, 75 y 100\%) de la SN no afectaron la variable número de frutos. Según Martínez et al. (2018) el efecto que causa el $\mathrm{Ca}\left(\mathrm{NO}_{3}\right)_{2}$ en concentraciones bajas consiste en generar una mayor producción de frutos por planta ya que a medida que se incrementa dicho fertilizante se pueden ocasionar rendimientos decrecientes. 
Cuadro 4. Comparación de medias de rendimiento y número de frutos en tres variedades de chile poblano.

\begin{tabular}{ccc}
\hline Concentración de la SN $(\%)$ & Rendimiento $\left(\mathrm{kg} \mathrm{planta}^{-1}\right)$ & Número de frutos por planta \\
\hline 50 & $1.2 \mathrm{a}$ & $24 \mathrm{a}$ \\
75 & $1.2 \mathrm{a}$ & $24.3 \mathrm{a}$ \\
100 & $1.1 \mathrm{a}$ & $21.9 \mathrm{a}$ \\
DHS & 0.1 & 2.9 \\
Variedad & $1.4 \mathrm{a}$ & \\
San Luis & $1 \mathrm{~b}$ & $19.8 \mathrm{~b}$ \\
Tlacotepec & $1 \mathrm{~b}$ & $24.2 \mathrm{a}$ \\
Tlalancaleca & 0.1 & $26.3 \mathrm{a}$ \\
DHS & 2.9
\end{tabular}

Medias con la misma letra en la misma columna son estadísticamente iguales (Tukey, $p \leq 0.05$ ); DHS= diferencia honesta significativa; $\mathrm{SN}=$ solución nutritiva.

\section{Calidad de fruto}

Las características de calidad de fruto de chile poblano no fueron afectadas por la concentración de la solución nutritiva, de acuerdo con los resultados de la prueba de comparación de medias, únicamente se presentaron diferencias significativas en la variedad San Luis (Cuadro 5). En las variedades criollas $100 \%$ de los frutos tuvo un peso entre los 80 y 110 g con la aplicación de cualquier concentración de la SN, mientras que en la variedad San Luis, el mayor número de frutos que se agruparon entre los 80 y $110 \mathrm{~g}$ se obtuvo al aplicar la SN al 75\%. Wamser et al. (2017). Indican que altas concentraciones de nitrógeno en la solución nutritiva aumentan la cantidad de frutos por planta debido a que es el principal componente que influye en el aumento del rendimiento de fruto; no obstante, a concentraciones elevadas en la solución nutritiva tiende a disminuir el peso de fruto a causa del aumento de la conductividad eléctrica en la solución del sustrato.

Cuadro 5. Caracterización de tamaño de fruto de tres variedades de chile poblano cultivadas con tres concentraciones de solución nutritiva.

\begin{tabular}{|c|c|c|c|c|}
\hline \multirow{2}{*}{ Peso (g) } & \multicolumn{3}{|c|}{ Concentración de la solución nutritiva (\%) } & \multirow{2}{*}{ DHS } \\
\hline & 50 & 75 & 100 & \\
\hline \multicolumn{5}{|c|}{ San Luis } \\
\hline Chico (80-110) & $9.42 \mathrm{ab}$ & $10.8 \mathrm{a}$ & $7 \mathrm{~b}$ & 3.2 \\
\hline Mediano (110-129.9) & $1.2 \mathrm{a}$ & $0.5 \mathrm{a}$ & $1.1 \mathrm{a}$ & 1 \\
\hline Grande $(130-150)$ & $0.2 \mathrm{a}$ & $0.3 \mathrm{a}$ & $0.4 \mathrm{a}$ & 0.5 \\
\hline Extra grande $(>150)$ & $0.1 \mathrm{a}$ & $0.1 \mathrm{a}$ & $0 \mathrm{a}$ & 0.2 \\
\hline \multicolumn{5}{|c|}{ Tlacotepec } \\
\hline \multicolumn{4}{|c|}{ Tlalancaleca } & 2.9 \\
\hline Chico (80-110) & $10.7 \mathrm{a}$ & $13.4 \mathrm{a}$ & $12.6 \mathrm{a}$ & 3.2 \\
\hline
\end{tabular}

Medias con la misma letra en la misma hilera son estadísticamente iguales (Tukey, $p \leq 0.05$ ); DHS= diferencia honesta significativa. 
Por otro lado, resultados similares presentaron Ramírez-Luna et al. (2005) quienes, en plantas de chile habanero producidas bajo invernadero, obtuvieron frutos de menor tamaño en comparación con las producidas en campo, debido a que las plantas recibieron una menor intensidad de luz, esta condición favoreció el desarrollo de plantas con mayor altura, tallos delgados, pero frutos de tamaño pequeño.

Referente al largo de fruto, hubo efectos significativos por la aplicación de la SN en las variedades SL y TC (Cuadro 6) en donde la solución nutritiva concentrada al $100 \%$ fomentó los frutos más largos (12-14 cm) en ambas variedades. San Juan et al. (2019) reportaron que en chile huacle obtuvieron una mayor respuesta en las variables largo y ancho de fruto con la aplicación de la solución nutritiva Steiner con una mayor concentración de nutrientes.

\begin{tabular}{|c|c|c|c|c|c|c|c|c|c|}
\hline \multirow{2}{*}{$\mathrm{FV}$} & \multirow{2}{*}{ GL } & \multicolumn{4}{|c|}{ Longitud $(\mathrm{cm})$} & \multicolumn{4}{|c|}{ Ancho $(\mathrm{cm})$} \\
\hline & & $\mathrm{CH}<10$ & MD 10-1.9 & GD 12-14 & $\mathrm{EG}>14$ & $\mathrm{CH}<6$ & MD 6-6.9 & GD 7-8 & $\mathrm{EG}>8$ \\
\hline \multicolumn{10}{|c|}{ San Luis } \\
\hline$\%$ de la SN & 2 & $11.1 \mathrm{~ns}$ & $31.4^{* *}$ & $3.6 \mathrm{~ns}$ & $4.7^{*}$ & $48.8^{* *}$ & $1.7 \mathrm{~ns}$ & $5.1 \mathrm{~ns}$ & $0.2 \mathrm{~ns}$ \\
\hline Error & 33 & 3.5 & 3.4 & 2.7 & 1.4 & 7.1 & 2.5 & 1.8 & 0.3 \\
\hline $\mathrm{CV}$ & & 102.6 & 57 & 43 & 81.6 & 63.5 & 45.6 & 57.7 & 142.7 \\
\hline \multicolumn{10}{|c|}{ Tlacotepec } \\
\hline$\%$ de la $\mathrm{SN}$ & 2 & $6 \mathrm{~ns}$ & $10 \mathrm{~ns}$ & $8.1 \mathrm{~ns}$ & $8.5^{*}$ & $41.7^{*}$ & $2.1 \mathrm{~ns}$ & $0.03 \mathrm{~ns}$ & - \\
\hline Error & 33 & 2.2 & 5.2 & 7 & 1.9 & 9.5 & 0.9 & 0.03 & - \\
\hline $\mathrm{CV}$ & & 67.1 & 53 & 65.7 & 135.4 & 28.2 & 159.5 & 600 & - \\
\hline \multicolumn{10}{|c|}{ Tlalancaleca } \\
\hline$\%$ de la SN & 2 & $2.1 \mathrm{~ns}$ & $0.4 \mathrm{~ns}$ & $2.2 \mathrm{~ns}$ & $5.4 \mathrm{~ns}$ & $39 \mathrm{~ns}$ & $1 \mathrm{~ns}$ & $1 \mathrm{~ns}$ & - \\
\hline Error & 33 & 2.9 & 6.9 & 5.3 & 2.6 & 15.1 & 2.3 & 0.6 & - \\
\hline $\mathrm{CV}$ & & 64.7 & 57.7 & 61.8 & 124.3 & 37.6 & 100.5 & 202.7 & - \\
\hline
\end{tabular}

$\mathrm{FV}=$ fuente de variación; $\mathrm{GL}=$ grados de libertad; $\mathrm{E}=$ error; $\mathrm{CV}=$ coeficiente de variación; $\mathrm{CH}=$ chico; $\mathrm{MD}=$ mediano; $\mathrm{GD}=$ grande; $\mathrm{EG}=$ extragrande $(\%) ; \mathrm{SN}=$ solución nutritiva; ${ }^{*}=$ significancia estadística al $0.05 ; \mathrm{ns}=$ no significativo.

En ancho de fruto tampoco hubo efecto de la SN, en la variedad SL la SN al 100\% causó el mayor porcentaje de frutos con un grosor entre 7 y $8 \mathrm{~cm}$, en la variedad TL más de $90 \%$ de los frutos presentaron un ancho inferior a los $6 \mathrm{~cm}$ con cualquier solución nutritiva, mientras que en la variedad TC la SN al 50\% propició el mayor número de frutos de tamaño mediano. Santiago et al. (2018) reportaron que los frutos de chile ancho poblano, cultivados a cielo abierto alcanzaron una longitud de $13.95 \mathrm{~cm}$ y diámetro de $6.25 \mathrm{~cm}$, valores que aunque no se obtuvieron bajo las mismas condiciones, son parecidos a los obtenidos en este trabajo en la variedad San Luis. 


\section{Conclusiones}

De las variedades estudiadas, el San Luis alcanzó el mayor rendimiento y calidad de fruto, pero fueron las variedades locales las que presentaron el mayor número de frutos, aunque con menor calidad. Sobre las concentraciones de las soluciones nutritivas estudiadas, no se presentaron diferencias estadísticas significativas en las variables más importantes como son rendimiento y número de frutos. El comportamiento agronómico de las variedades criollas evaluadas en el sistema de sustrato, invernadero e hidroponía es comparable con la variedad San Luis y puede ser una opción para la producción de chile poblano en la región del Alto Atoyac.

\section{Literatura citada}

Aguilar, A. J. L.; Grageda, C. O. A.; Vuelvas, C. M. A.; Martínez, H. M.; Solís, M. E.; Medina, C. T. y Ramírez, R. A. 2005. Eficiencia de fertilizantes aplicados con fertirriego en chile ancho (Capsicum annuum L.). México. Agric. Téc. Méx. 31(2):177-189.

Aguirre, H. E. y Muñoz O. V. 2015. El chile como alimento. México. Rev. Acad. Mex. Cienc. AC. 66(3):16-23.

Alcántar-González, G.; Trejo-Téllez, L. I. y Gómez-Merino. F. C. 2016. Nutrición de cultivos. Colegio de postgraduados. Biblioteca básica de agricultura. ISBN 978-607-715-324-5. México, DF. 443 p.

Alemán, P. R. D.; Domínguez, B. J.; Rodríguez, G. Y.; Soria R. S.; Torres, G. R.; Vargas, B. J. C.; Bravo, M. C. y Alba, R. J. L. 2018. Indicadores morfofisiológicos y productivos del pimiento sembrado en invernadero y a campo abierto en las condiciones de la amazonía ecuatoriana. Cuba. Centro Agrícola. 45(1):14-23.

Beltrán-Morales, F. A.; García-Hernández, J. L.; Ruiz-Espinoza, F. H.; Valdez-Cepeda, R. D.; Preciado-Rangel, P.; Fortis-Hernández, M. y González-Zamora, A. 2016. Efecto de sustratos orgánicos en el crecimiento de seis variedades de chile jalapeño (Capsicum аппиит L.). México. Ecosistemas y Recursos Agropecuarios. 3(7):143-149.

Berrospe-Ochoa, E. A.; Saucedo-Veloz, C.; Ramírez-Vallejo, P. y Ramírez-Guzmán, M. E. 2015. Comportamiento agronómico de plántulas de poblaciones nativas de jitomate (Solanum lycopersicum L.) en producción intensiva en invernadero. Agrociencia. 49(6):637-650.

Elizondo-Cabalceta, E. y Monge-Pérez, J. E. 2017. Caracterización morfológica de 15 genotipos de pimiento (Capsicum annuum) cultivados bajo invernadero en Costa Rica. Costa Rica. InterSedes. 18(37):129-154. https://doi.org/10.15517/isucr.v18i37.28652.

Fawzy, Z. F.; El-Bassiony, A. M.; Yunsheng, L.; Zhu, O. and Ghoname, A. A. 2012. Effect of mineral, organic and bio-N fertilizers on growth, yield and fruit quality of sweet pepper. Jordania J. Appl. Sci. Res. 8(8):3921-3933.

González-Pérez, E.; Yáñez-Morales, M. J.; Santiago-Santiago, V. y Montero-Pineda, Á. 2004. Biodiversidad fungosa en la marchitez del chile y algunos factores involucrados, en Tlacotepec de José Manzo, el Verde, Puebla. Agrociencia. 38(6):653-661.

Hernández-Verdugo, S.; González-Sánchez, R. A.; Porras, F.; Parra-Terraza, S.; Valdez-Ortiz, A.; Pacheco-Olvera, A. y López-España, R. G. 2015. Plasticidad fenotípica de poblaciones de chile silvestre (Capsicum annuиm var. Glabriusculum) en respuesta a disponibilidad de luz. México. Bot. Sci. 93(2):1-10. https://doi.org/10.17129/botsci.237. 
Hunt, R. 2017. Growth analysis, individual plants. Encyclopedia of applied plant sciences, second edition. Academic press, elsevier Ltd. Amsterdam, netherlands. ISBN: 9780123948083. 421-429. http://dx.doi.org/10.1016/B978-0-12-394807-6.00226-4.

INEGI. 2010. Instituto Nacional de Estadística y Geografía. Censo de población y vivienda 2010. México.

López-Gómez, J. D.; Villegas-Torres, O. G.; Sotelo-Nava, H.; Andrade-Rodríguez, M.; JuárezLópez, P. y Martínez-Fernández, E. 2017.Rendimiento y calidad del chile habanero (Capsicum chinense Jacq.) por efecto del régimen nutrimental. México. Rev. Mex. Cienc. Agríc. 8(8):1747-1758. https://doi.org/10.29312/remexca.v8i8.699.

Luna-Fletes, J. A.; Can-Chulim, Á.; Cruz-Crespo, E.; Bugarín-Montoya, R., y Valdivia-Reynoso, M. G. 2018. Intensidad de raleo y soluciones nutritivas en la calidad de tomate cherry. Rev. Fitotec. Mex. 41(1):57-66.

Magdaleno-Villar, J. J.; Peña-Lomelí, A.; Castro-Brindis, R.; Castillo-González, A. M.; GalvisSpinola, A., Ramírez-Pérez, F. y Hernández-Hernández, B. 2006. Efecto de soluciones nutritivas sobre el desarrollo de plántulas de tomate de cáscara (Physalis ixocarpa Brot.). Rev. Chapingo Ser. Hortic. 12(2):223-229.

Martínez, M. L.; Velasco, V. V. A.; Ruiz, L. J.; Enríquez-del Valle, J. R.; Campos, Á. G. V. y Montaño, L. M. L. 2018. Efecto del nitrato de calcio y sustratos en el rendimiento del tomate. México. Rev. Mex. Cienc. Agríc. 4(spe. 6):1175-1184. https://doi.org/10.29312/ remexca.v0i6.1281.

Mendoza-Pérez, C.; Ramírez-Ayala, C.; Ojeda-Bustamante, W. and Flores-Magdaleno, H. 2017. Estimation of leaf area index and yield of greenhouse-grown poblano pepper. Ingeniería Agrícola y Biosistemas. 9(1):37-50. https://doi.org/10.5154/r.inagbi.2017.04.009.

Moreno, P. E. C.; Mora, A. R.; Sánchez, C. F. y García-Pérez, V. 2011. Fenología y rendimiento de híbridos de pimiento morrón (Capsicum annuum L.) cultivados en hidroponía. Rev. Chapingo Ser. Hortic. 17(spe. 2):5-18.

Moreno, R. A.; Rodríguez, D. N.; Reyes, C. J. L.; Quiroz, M. C. y Reyes, J. G. 2014. Comportamiento del chile húngaro (Capsicum annuum) en mezclas de vermicompostarena bajo condiciones protegidas. Universidad Nacional de Cuyo. Rev. Facult. Cienc. Agrar. 46(2):97-111.

Norma Mexicana. 2014. NMX-FF-025-SCFI-2014 Productos alimenticios no industrializados para consumo humano - chile fresco (Capsicum spp.) Especificaciones. Dirección General de Normas. Secretaría de Economía. 31 p.

Pérez, C. L. J.; Tornero, C. M. A.; Escobedo, G. J. S. y Sandoval, C. E. 2017. El chile poblano criollo en la cultura alimentaria del Alto Atoyac. México. Estudios Sociales. 27(49):47-66.

Ponce, V. J. J.; Peña-Lomelí, A.; Rodríguez-Pérez, J. E.; Mora-Aguilar, R.; Castro-Brindis, R. y Magaña, L. N. 2012. Densidad y poda en tres variedades de tomate de cáscara (Physalis ixocarpa Brot. ex Horm.) cultivado en invernadero. Rev. Chapingo Ser. Hortic. 18(3):325332. http://dx.doi.org/10.5154/r.rchsh.2010.08.028.

Ramírez-Luna, E.; Castillo-Aguilar, C. de la C.; Aceves-Navarro, E. y Carrillo-Ávila, E. 2005. Efecto de productos con reguladores de crecimiento sobre la floración y amarre de fruto en chile 'habanero'. Rev. Chapingo Ser. Hortic. 11(1):93-98.

Ramos-Gourcy, F. y De Luna-Jiménez, A. 2006. Evaluación de tres variedades de chile (Capsicum annuит L.) en cuatro concentraciones de una solución hidropónica bajo invernadero. México. Investigación y Ciencia. 14(34):6-11. 
Reséndiz-Melgar, R. C.; Moreno-Pérez, E. C.; Sánchez-del Castillo, F.; Rodríguez-Pérez, J. E. y Peña-Lomelí, A. 2010. Variedades de pimiento morrón manejadas con despunte temprano en dos densidades de población. Rev. Chapingo Ser. Hortic. 16(3):223-229.

Rodríguez, J.; Peña, O. B. V.; Gil, M. A.; Martínez, C. B.; Manzo, F. and Salazar, L. 2007. Rescate in situ del chile 'poblano' en Puebla, México. Rev. Fitotec. Mex. 30(1):25-32.

San Juan, M. J.; Aquino-Bolaños, T.; Ortiz-Hernández, Y. D. y Cruz-Izquierdo, S. 2019. Características de fruto y semilla de chile huacle (Capsicum annuum L.) producido en hidroponía. Chile. Idesia Arica. 37(2):87-94. https://dx.doi.org/10.4067/S071834292019000200087.

San Martín-Hernández, C.; Ordaz-Chaparro, V. M.; Sánchez-García, P.; Colinas-Leon, M. T. and Borges-Gómez, L. 2012. Tomato (Solanum lycopersicum L.) quality produced in hydroponics with different particle sizes of tezontle. Agrociencia. 46(3):243-254.

Sánchez-del-Castillo, F.; González-Molina, L.; Moreno-Pérez, E. del C.; Pineda-Pineda, J. y Reyes-González, C. E. 2014. Dinámica nutrimental y rendimiento de pepino cultivado en hidroponía con y sin recirculación de la solución nutritiva. México. Rev. Fitotec. Mex. 37(3):261-269.

Santiago, L. U.; Ramírez, M. M. y Méndez, A. R. 2018. HAP14F: híbrido de chile ancho poblano para el Altiplano de México. México. Rev. Mex. Cienc. Agríc. 9(2):481-485. https://doi.org/10.29312/remexca.v9i2.1088.

SIAP. 2019. Servicio de Información Agroalimentaria y Pesquera. Panorama agroalimentario. México. 218 p.

SAS. 2002. Statistical analysis System, Institute. SAS User's Guide version 9.0. Cary NC. USA. $44 \mathrm{p}$.

Steiner, A. A. 1984. The universal nutrient solution. Proceeding of the Sixth. International congress on soilless culture, lunteren. Netherlands. ISOSC. 633-649 p.

Toledo-Aguilar, R.; López-Sánchez, H.; Antonio, L. P.; Guerrero-Rodríguez, J. D.; SantacruzVarela, A. and Huerta-de la Peña, A. 2011. Vegetative, reproductive and fruit yield characteristics of 'poblano' pepper landraces. Rev. Chapingo Ser. Hortic. 17(3):139-150.

Velasco, H. E.; Nieto, A. R. y Navarro, L. E. R. 2011. Cultivo de tomate en hidroponía e invernadero. Colegio de postgraduados, Mundi-Prensa. México, DF. 126 p.

Velásquez-Valle, R.; Reveles-Torres, L. R.; Reveles-Hernández, M.; Cid-Ríos, J. Á. y MauricioCastillo, J. A. 2014. Rendimiento y calidad de fruto de cuatro líneas de chile ancho en Zacatecas, México, DF. Agrofaz. 14(3):85.92.

Wamser, A. F.; Cecilio, F. A. B.; Nowaki. R. H. D.; Mendoza-Cortez, J. W. and Urrestarazu, M. 2017. Influence of drainage and nutrient-solution nitrogen and potassium concentrations on the agronomic behavior of bell-pepper plants cultivated in a substrate. US. Plos One. 12(7):e0180529. https://doi.org/10.1371/journal.pone.0180529. 